Acta Crystallographica Section E

Structure Reports

Online

ISSN 1600-5368

\section{2,4-Dioxo-1-(prop-2-ynyl)-1,2,3,4-tetra- hydropyrimidine-5-carbaldehyde}

\section{Yan He, Liang-Yan Cui and Xin-Ying Zhang*}

School of Chemistry and Environmental Science, Henan Key Laboratory for Environmental Pollution Control, Henan Normal University, Xinxiang, Henan 453007, People's Republic of China

Correspondence e-mail: xyzh518@sohu.com

Received 3 August 2011; accepted 9 August 2011

Key indicators: single-crystal X-ray study; $T=296 \mathrm{~K}$; mean $\sigma(\mathrm{C}-\mathrm{C})=0.002 \AA$; $R$ factor $=0.040 ; w R$ factor $=0.123 ;$ data-to-parameter ratio $=12.9$.

In the crystal structure of the title compound, $\mathrm{C}_{8} \mathrm{H}_{6} \mathrm{~N}_{2} \mathrm{O}_{3}$, the molecules are linked by a pairs of intermolecular $\mathrm{N}-\mathrm{H} \cdots \mathrm{O}$ hydrogen bonds, forming inversion dimers. The aldehyde group is in the same plane as the pyrimidine ring [with a maximum deviation of 0.083 (2) $\AA$ for the $\mathrm{O}$ atom), and the linear propargyl group $\left[\mathrm{C}-\mathrm{C}-\mathrm{C}=178.99(19)^{\circ}\right]$ makes a dihedral angle of $74.36(13)^{\circ}$ with the ring.

\section{Related literature}

For applications of acyclic pyrimidine nucleosides, see: De Clercq (2009, 2010a,b); Fan et al. (2011).

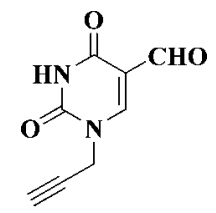

\section{Experimental}

\section{Crystal data}

$\mathrm{C}_{8} \mathrm{H}_{6} \mathrm{~N}_{2} \mathrm{O}_{3}$

$M_{r}=178.15$

Monoclinic, $P 2_{1} / n$

$a=5.1756(7) \AA$

$$
\begin{aligned}
& Z=4 \\
& \text { Mo } K \alpha \text { radiation } \\
& \mu=0.11 \mathrm{~mm}^{-1}
\end{aligned}
$$

Data collection

Bruker SMART CCD area-detector diffractometer

Absorption correction: multi-scan (SADABS; Bruker, 1997)

$T_{\min }=0.955, T_{\max }=0.972$

Refinement

$R\left[F^{2}>2 \sigma\left(F^{2}\right)\right]=0.040$

$w R\left(F^{2}\right)=0.123$

$S=1.08$

1520 reflections

$T=296 \mathrm{~K}$

$0.41 \times 0.37 \times 0.25 \mathrm{~mm}$

5826 measured reflections 1520 independent reflections 1261 reflections with $I>2 \sigma(I)$ $R_{\text {int }}=0.020$

\section{Table 1}

Hydrogen-bond geometry $\left(\AA{ }^{\circ}\right)$.

\begin{tabular}{lllll}
\hline$D-\mathrm{H} \cdots A$ & $D-\mathrm{H}$ & $\mathrm{H} \cdots A$ & $D \cdots A$ & $D-\mathrm{H} \cdots A$ \\
\hline $\mathrm{N} 2-\mathrm{H} 2 \cdots \mathrm{O}^{\mathrm{i}}$ & 0.86 & 1.98 & $2.8329(18)$ & 174 \\
\hline
\end{tabular}

Symmetry code: (i) $-x+2,-y+2,-z+1$.

Data collection: SMART (Bruker, 1997); cell refinement: SAINT (Bruker, 1997); data reduction: SAINT; program(s) used to solve structure: SHELXS97 (Sheldrick, 2008); program(s) used to refine structure: SHELXL97 (Sheldrick, 2008); molecular graphics: SHELXTL (Sheldrick, 2008); software used to prepare material for publication: SHELXTL.

This work was supported by the National Natural Science Foundation of China (No. 20972042) and the Natural Science Foundation of Department of Education of Henan Province (No. 2008 A150013).

Supplementary data and figures for this paper are available from the IUCr electronic archives (Reference: IS2760).

\section{References}

Bruker (1997). SADABS, SMART and SAINT. Bruker AXS Inc., Madison, Wisconsin, USA.

De Clercq, E. (2009). Rev. Med. Virol. 19, 287-299.

De Clercq, E. (2010a). J. Med. Chem. 53, 1438-1450.

De Clercq, E. (2010b). Antiviral Res. 85, 19-24.

Fan, X.-S., Wang, Y.-Y., Qu, Y.-Y., Xu, H.-Y., He, Y., Zhang, X.-Y. \& Wang, J.-J. (2011). J. Org. Chem. 76, 982-985.

Sheldrick, G. M. (2008). Acta Cryst. A64, 112-122. 


\section{supporting information}

Acta Cryst. (2011). E67, o2350 [doi:10.1107/S1600536811032272]

\section{2,4-Dioxo-1-(prop-2-ynyl)-1,2,3,4-tetrahydropyrimidine-5-carbaldehyde}

\section{Yan He, Liang-Yan Cui and Xin-Ying Zhang}

\section{S1. Comment}

Acyclic pyrimidine nucleosides have drawn much attention because of their insteresting structures and broad utilizations as effective drugs for the treatment of diseases caused by herpes simplex virus (HSV) and varizella zoster (VZV) (De Clercq, 2009, 2010a,b). The title compound can be used as a powerful synthon for the preparation of acyclic pyrimidine nucleoside derivatives with potential biological activities due to the rich and extensive chemistry of the aldehyde carbonyl (Fan, 2011). Herein, we report the synthesis and crystal structure of the title compound.

In the title compound, $\mathrm{C}_{8} \mathrm{H}_{6} \mathrm{~N}_{2} \mathrm{O}_{3}$, all the atoms in the pyrimidine ring, atoms connected directly with the pyrimidine ring and atoms in the aldehyde carbonyl group in the 5-position of the pyrimidine ring are in the same plane, which means there is a big conjugated system in the molecule. The linear structure of the propynyl group is connected with the big plane at an angle of $150.3^{\circ}$. In the crystal structure, the molecules are linked via intermolecular $\mathrm{N}-\mathrm{H} \cdots \mathrm{O}$ hydrogen bond.

\section{S2. Experimental}

To a solution of $\mathrm{K}_{2} \mathrm{~S}_{2} \mathrm{O}_{8}(16.5 \mathrm{mmol})$ and $\mathrm{CuSO}_{4}(3.2 \mathrm{mmol})$ in $30 \mathrm{ml} \mathrm{H}_{2} \mathrm{O}$ was added a $\mathrm{CH}_{3} \mathrm{CN}$ solution $(25 \mathrm{ml})$ of 5 methyl-1-(prop-2-ynyl)pyrimidine-2,4(1H,3H)-dione $(8 \mathrm{mmol})$ and 2,6-lutidine $(3.2 \mathrm{ml})$. The mixture was stirred at 60 ${ }^{\circ} \mathrm{C}$ for $5 \mathrm{~h}$. Upon completion, the mixture was concentrated to half of the initial volume, and the remaining solution was extracted with EtOAc. The organic layer was washed with $\mathrm{H}_{2} \mathrm{O}$. The aqueous layers were combined and back-extracted with $\mathrm{CHCl}_{3}$. Then the organic layers were combined, dried over $\mathrm{Na}_{2} \mathrm{SO}_{4}$, and then concentrated. The residue was purified through silica gel column chromatography with a mixture of methylene chloride-methanol $(60: 1, v / v)$ as eluent to give 1,2,3,4-tetrahydro-2,4-dioxo-1-(prop-2-ynyl)- pyrimidine-5-carbaldehyde. Single crystals of the title compound were obtained by slow evaporation of the solvent from a methylene chloride-methanol $(60: 1 \mathrm{v} / \mathrm{v})$ solution.

\section{S3. Refinement}

$\mathrm{H}$ atoms were positioned geometrically and refined using riding model, with $\mathrm{C}-\mathrm{H}=0.93$ or $0.97 \AA$, and $\mathrm{N}-\mathrm{H}=0.86 \AA$, and with $U_{\text {iso }}(\mathrm{H})=1.2 U_{\mathrm{eq}}(\mathrm{C}, \mathrm{N})$. 


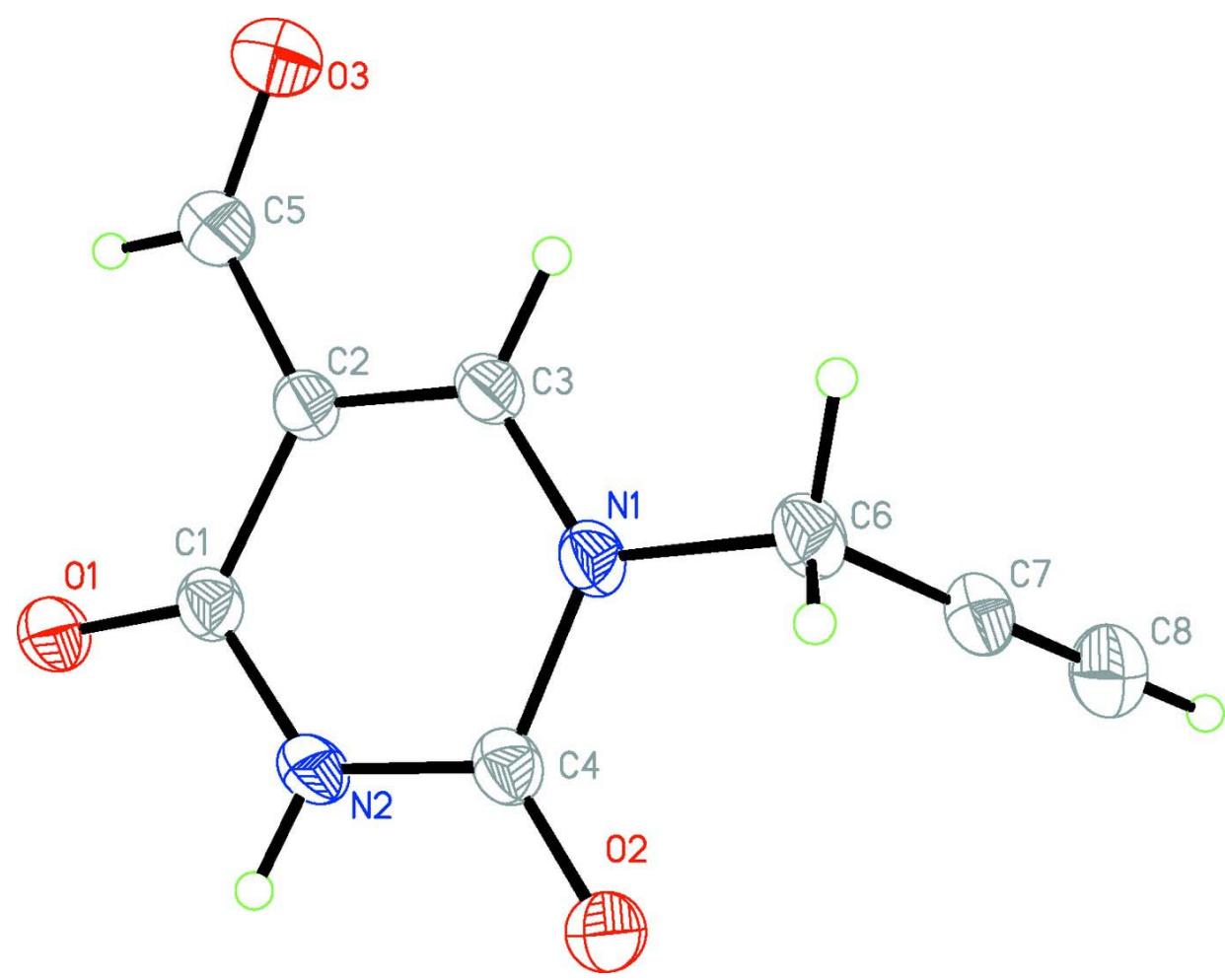

\section{Figure 1}

Molecular structure of the title compound, with displacement ellipsoids drawn at the $30 \%$ probability level.

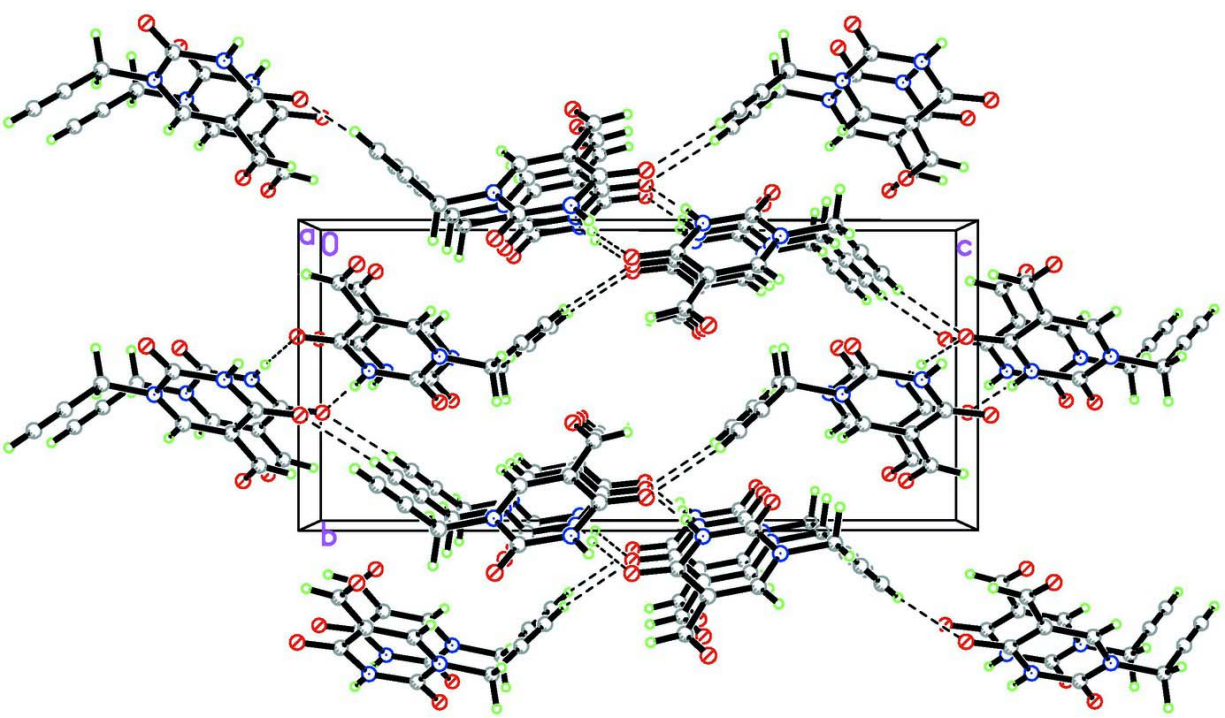

\section{Figure 2}

Crystal packing of the title compound with view along the $a$ axis. Intermolecular $\mathrm{N}-\mathrm{H} \cdots \mathrm{O}$ hydrogen bonds are shown as dashed lines. 


\section{2,4-Dioxo-1-(prop-2-ynyl)-1,2,3,4-tetrahydropyrimidine-5-carbaldehyde}

Crystal data

$$
\begin{aligned}
& \mathrm{C}_{8} \mathrm{H}_{6} \mathrm{~N}_{2} \mathrm{O}_{3} \\
& M_{r}=178.15 \\
& \text { Monoclinic, } P 2_{1} / n \\
& \text { Hall symbol: }-\mathrm{P} 2 \mathrm{yn} \\
& a=5.1756(7) \AA \\
& b=8.4877(12) \AA \\
& c=18.565(3) \AA \\
& \beta=90.611(2)^{\circ} \\
& V=815.5(2) \AA^{3} \\
& Z=4
\end{aligned}
$$

\section{Data collection}

Bruker SMART CCD area-detector diffractometer

Radiation source: fine-focus sealed tube Graphite monochromator phi and $\omega$ scans Absorption correction: multi-scan

(SADABS; Bruker, 1997)

$T_{\min }=0.955, T_{\max }=0.972$

\section{Refinement}

Refinement on $F^{2}$

Least-squares matrix: full

$R\left[F^{2}>2 \sigma\left(F^{2}\right)\right]=0.040$

$w R\left(F^{2}\right)=0.123$

$S=1.08$

1520 reflections

118 parameters

0 restraints

Primary atom site location: structure-invariant direct methods

$$
F(000)=368
$$

$D_{\mathrm{x}}=1.451 \mathrm{Mg} \mathrm{m}^{-3}$

Mo $K \alpha$ radiation, $\lambda=0.71073 \AA$

Cell parameters from 2188 reflections

$\theta=2.6-26.7^{\circ}$

$\mu=0.11 \mathrm{~mm}^{-1}$

$T=296 \mathrm{~K}$

Block, colourless

$0.41 \times 0.37 \times 0.25 \mathrm{~mm}$

5826 measured reflections

1520 independent reflections

1261 reflections with $I>2 \sigma(I)$

$R_{\text {int }}=0.020$

$\theta_{\text {max }}=25.5^{\circ}, \theta_{\min }=2.6^{\circ}$

$h=-6 \rightarrow 6$

$k=-10 \rightarrow 10$

$l=-22 \rightarrow 21$

Secondary atom site location: difference Fourier map

Hydrogen site location: inferred from

neighbouring sites

$\mathrm{H}$-atom parameters constrained

$w=1 /\left[\sigma^{2}\left(F_{\mathrm{o}}^{2}\right)+(0.0694 P)^{2}+0.1695 P\right]$

where $P=\left(F_{\mathrm{o}}^{2}+2 F_{\mathrm{c}}^{2}\right) / 3$

$(\Delta / \sigma)_{\max }<0.001$

$\Delta \rho_{\max }=0.14$ e $\AA^{-3}$

$\Delta \rho_{\min }=-0.23$ e $\AA^{-3}$

\section{Special details}

Geometry. All e.s.d.'s (except the e.s.d. in the dihedral angle between two 1.s. planes)

are estimated using the full covariance matrix. The cell e.s.d.'s are taken into account individually in the estimation of e.s.d.'s in distances, angles and torsion angles; correlations between e.s.d.'s in cell parameters are only used when they are defined by crystal symmetry. An approximate (isotropic) treatment of cell e.s.d.'s is used for estimating e.s.d.'s involving 1.s. planes.

Refinement. Refinement of $F^{2}$ against ALL reflections. The weighted $R$-factor $w R$ and goodness of fit $S$ are based on $F^{2}$, conventional $R$-factors $R$ are based on $F$, with $F$ set to zero for negative $F^{2}$. The threshold expression of $F^{2}>\sigma\left(F^{2}\right)$ is used only for calculating $R$-factors(gt) $e t c$. and is not relevant to the choice of reflections for refinement. $R$-factors based on $F^{2}$ are statistically about twice as large as those based on $F$, and $R$ factors based on ALL data will be even larger. 
Fractional atomic coordinates and isotropic or equivalent isotropic displacement parameters $\left(\AA^{2}\right)$

\begin{tabular}{lllll}
\hline & $x$ & $y$ & $z$ & $U_{\text {iso }}^{*} / U_{\text {eq }}$ \\
\hline C1 & $0.7042(3)$ & $0.8893(2)$ & $0.44458(8)$ & $0.0374(4)$ \\
C2 & $0.5046(3)$ & $0.8128(2)$ & $0.40196(8)$ & $0.0376(4)$ \\
C3 & $0.4934(3)$ & $0.84333(19)$ & $0.33033(8)$ & $0.0372(4)$ \\
H3 & 0.3631 & 0.7959 & 0.3031 & $0.045^{*}$ \\
C4 & $0.8652(3)$ & $1.01190(19)$ & $0.33360(8)$ & $0.0368(4)$ \\
C5 & $0.3180(4)$ & $0.7061(2)$ & $0.43522(10)$ & $0.0510(5)$ \\
H5 & 0.3435 & 0.6787 & 0.4833 & $0.061^{*}$ \\
C6 & $0.6377(3)$ & $0.9724(2)$ & $0.21870(8)$ & $0.0432(4)$ \\
H6A & 0.4685 & 0.9390 & 0.2017 & $0.052^{*}$ \\
H6B & 0.6512 & 1.0851 & 0.2110 & $0.052^{*}$ \\
C7 & $0.8363(4)$ & $0.8923(2)$ & $0.17686(9)$ & $0.0464(5)$ \\
C8 & $0.9931(4)$ & $0.8282(3)$ & $0.14242(11)$ & $0.0612(6)$ \\
H8 & 1.1175 & 0.7773 & 0.1151 & $0.073^{*}$ \\
N1 & $0.6616(2)$ & $0.93903(17)$ & $0.29646(7)$ & $0.0370(4)$ \\
N2 & $0.8664(3)$ & $0.98582(16)$ & $0.40649(7)$ & $0.0398(4)$ \\
H2 & 0.9818 & 1.0356 & 0.4312 & $0.048^{*}$ \\
O1 & $0.7344(2)$ & $0.87340(16)$ & $0.51012(6)$ & $0.0490(4)$ \\
O2 & $1.0262(2)$ & $1.09061(15)$ & $0.30334(6)$ & $0.0472(4)$ \\
O3 & $0.1324(3)$ & $0.65141(19)$ & $0.40376(8)$ & $0.0685(5)$ \\
\end{tabular}

Atomic displacement parameters $\left(\AA^{2}\right)$

\begin{tabular}{lllllll}
\hline & $U^{11}$ & $U^{22}$ & $U^{33}$ & $U^{12}$ & $U^{13}$ & $U^{23}$ \\
\hline C1 & $0.0360(8)$ & $0.0454(9)$ & $0.0308(8)$ & $0.0027(7)$ & $-0.0031(6)$ & $-0.0023(7)$ \\
C2 & $0.0353(8)$ & $0.0442(9)$ & $0.0333(8)$ & $0.0011(7)$ & $-0.0018(6)$ & $-0.0066(7)$ \\
C3 & $0.0326(8)$ & $0.0439(9)$ & $0.0350(8)$ & $0.0021(7)$ & $-0.0041(6)$ & $-0.0089(7)$ \\
C4 & $0.0365(8)$ & $0.0414(9)$ & $0.0323(8)$ & $0.0027(7)$ & $-0.0036(7)$ & $-0.0023(7)$ \\
C5 & $0.0503(10)$ & $0.0600(11)$ & $0.0427(10)$ & $-0.0103(9)$ & $-0.0011(8)$ & $-0.0042(8)$ \\
C6 & $0.0451(10)$ & $0.0551(10)$ & $0.0294(8)$ & $0.0008(8)$ & $-0.0085(7)$ & $0.0013(7)$ \\
C7 & $0.0533(11)$ & $0.0548(11)$ & $0.0311(8)$ & $-0.0078(9)$ & $-0.0027(8)$ & $-0.0019(8)$ \\
C8 & $0.0625(13)$ & $0.0738(14)$ & $0.0474(11)$ & $-0.0033(11)$ & $0.0076(10)$ & $-0.0125(10)$ \\
N1 & $0.0366(7)$ & $0.0471(8)$ & $0.0272(7)$ & $0.0016(6)$ & $-0.0044(5)$ & $-0.0029(6)$ \\
N2 & $0.0402(8)$ & $0.0494(8)$ & $0.0297(7)$ & $-0.0079(6)$ & $-0.0084(5)$ & $-0.0014(6)$ \\
O1 & $0.0500(7)$ & $0.0683(8)$ & $0.0285(6)$ & $-0.0120(6)$ & $-0.0057(5)$ & $0.0017(5)$ \\
O2 & $0.0470(7)$ & $0.0566(8)$ & $0.0378(7)$ & $-0.0094(6)$ & $-0.0019(5)$ & $0.0039(5)$ \\
O3 & $0.0592(9)$ & $0.0805(11)$ & $0.0658(10)$ & $-0.0216(7)$ & $0.0004(7)$ & $-0.0139(8)$ \\
& & & & & &
\end{tabular}

Geometric parameters $\left(A,{ }^{\circ}\right)$

\begin{tabular}{llll}
\hline $\mathrm{C} 1-\mathrm{O} 1$ & $1.2325(19)$ & $\mathrm{C} 5-\mathrm{O} 3$ & $1.211(2)$ \\
$\mathrm{C} 1-\mathrm{N} 2$ & $1.374(2)$ & $\mathrm{C} 5-\mathrm{H} 5$ & 0.9300 \\
$\mathrm{C} 1-\mathrm{C} 2$ & $1.449(2)$ & $\mathrm{C} 6-\mathrm{C} 7$ & $1.462(3)$ \\
$\mathrm{C} 2-\mathrm{C} 3$ & $1.356(2)$ & $\mathrm{C} 6-\mathrm{N} 1$ & $1.475(2)$ \\
$\mathrm{C} 2-\mathrm{C} 5$ & $1.465(3)$ & $\mathrm{C} 6-\mathrm{H} 6 \mathrm{~A}$ & 0.9700 \\
$\mathrm{C} 3-\mathrm{N} 1$ & $1.351(2)$ & $\mathrm{C} 6-\mathrm{H} 6 \mathrm{~B}$ & 0.9700
\end{tabular}




\begin{tabular}{|c|c|c|c|}
\hline $\mathrm{C} 3-\mathrm{H} 3$ & 0.9300 & $\mathrm{C} 7-\mathrm{C} 8$ & $1.173(3)$ \\
\hline $\mathrm{C} 4-\mathrm{O} 2$ & $1.211(2)$ & $\mathrm{C} 8-\mathrm{H} 8$ & 0.9300 \\
\hline $\mathrm{C} 4-\mathrm{N} 2$ & $1.371(2)$ & $\mathrm{N} 2-\mathrm{H} 2$ & 0.8600 \\
\hline $\mathrm{C} 4-\mathrm{N} 1$ & $1.397(2)$ & & \\
\hline $\mathrm{O} 1-\mathrm{C} 1-\mathrm{N} 2$ & $120.11(14)$ & $\mathrm{C} 7-\mathrm{C} 6-\mathrm{N} 1$ & $112.24(14)$ \\
\hline $\mathrm{O} 1-\mathrm{C} 1-\mathrm{C} 2$ & $124.91(15)$ & $\mathrm{C} 7-\mathrm{C} 6-\mathrm{H} 6 \mathrm{~A}$ & 109.2 \\
\hline $\mathrm{N} 2-\mathrm{C} 1-\mathrm{C} 2$ & $114.98(13)$ & $\mathrm{N} 1-\mathrm{C} 6-\mathrm{H} 6 \mathrm{~A}$ & 109.2 \\
\hline $\mathrm{C} 3-\mathrm{C} 2-\mathrm{C} 1$ & $118.22(15)$ & $\mathrm{C} 7-\mathrm{C} 6-\mathrm{H} 6 \mathrm{~B}$ & 109.2 \\
\hline $\mathrm{C} 3-\mathrm{C} 2-\mathrm{C} 5$ & $120.68(15)$ & $\mathrm{N} 1-\mathrm{C} 6-\mathrm{H} 6 \mathrm{~B}$ & 109.2 \\
\hline $\mathrm{C} 1-\mathrm{C} 2-\mathrm{C} 5$ & $121.10(15)$ & $\mathrm{H} 6 \mathrm{~A}-\mathrm{C} 6-\mathrm{H} 6 \mathrm{~B}$ & 107.9 \\
\hline $\mathrm{N} 1-\mathrm{C} 3-\mathrm{C} 2$ & $123.42(14)$ & $\mathrm{C} 8-\mathrm{C} 7-\mathrm{C} 6$ & $178.99(19)$ \\
\hline $\mathrm{N} 1-\mathrm{C} 3-\mathrm{H} 3$ & 118.3 & $\mathrm{C} 7-\mathrm{C} 8-\mathrm{H} 8$ & 180.0 \\
\hline $\mathrm{C} 2-\mathrm{C} 3-\mathrm{H} 3$ & 118.3 & $\mathrm{C} 3-\mathrm{N} 1-\mathrm{C} 4$ & $121.52(13)$ \\
\hline $\mathrm{O} 2-\mathrm{C} 4-\mathrm{N} 2$ & $123.44(14)$ & $\mathrm{C} 3-\mathrm{N} 1-\mathrm{C} 6$ & $121.56(13)$ \\
\hline $\mathrm{O} 2-\mathrm{C} 4-\mathrm{N} 1$ & $122.30(14)$ & $\mathrm{C} 4-\mathrm{N} 1-\mathrm{C} 6$ & $116.91(14)$ \\
\hline $\mathrm{N} 2-\mathrm{C} 4-\mathrm{N} 1$ & $114.26(14)$ & $\mathrm{C} 4-\mathrm{N} 2-\mathrm{C} 1$ & $127.43(13)$ \\
\hline $\mathrm{O} 3-\mathrm{C} 5-\mathrm{C} 2$ & $123.80(18)$ & $\mathrm{C} 4-\mathrm{N} 2-\mathrm{H} 2$ & 116.3 \\
\hline $\mathrm{O} 3-\mathrm{C} 5-\mathrm{H} 5$ & 118.1 & $\mathrm{C} 1-\mathrm{N} 2-\mathrm{H} 2$ & 116.3 \\
\hline $\mathrm{C} 2-\mathrm{C} 5-\mathrm{H} 5$ & 118.1 & & \\
\hline $\mathrm{O} 1-\mathrm{C} 1-\mathrm{C} 2-\mathrm{C} 3$ & $179.16(16)$ & $\mathrm{O} 2-\mathrm{C} 4-\mathrm{N} 1-\mathrm{C} 3$ & $175.63(15)$ \\
\hline $\mathrm{N} 2-\mathrm{C} 1-\mathrm{C} 2-\mathrm{C} 3$ & $-0.7(2)$ & $\mathrm{N} 2-\mathrm{C} 4-\mathrm{N} 1-\mathrm{C} 3$ & $-4.1(2)$ \\
\hline $\mathrm{O} 1-\mathrm{C} 1-\mathrm{C} 2-\mathrm{C} 5$ & $-0.4(3)$ & $\mathrm{O} 2-\mathrm{C} 4-\mathrm{N} 1-\mathrm{C} 6$ & $-4.8(2)$ \\
\hline $\mathrm{N} 2-\mathrm{C} 1-\mathrm{C} 2-\mathrm{C} 5$ & $179.81(15)$ & $\mathrm{N} 2-\mathrm{C} 4-\mathrm{N} 1-\mathrm{C} 6$ & $175.44(14)$ \\
\hline $\mathrm{C} 1-\mathrm{C} 2-\mathrm{C} 3-\mathrm{N} 1$ & $1.3(2)$ & $\mathrm{C} 7-\mathrm{C} 6-\mathrm{N} 1-\mathrm{C} 3$ & $-106.92(18)$ \\
\hline $\mathrm{C} 5-\mathrm{C} 2-\mathrm{C} 3-\mathrm{N} 1$ & $-179.19(15)$ & $\mathrm{C} 7-\mathrm{C} 6-\mathrm{N} 1-\mathrm{C} 4$ & $73.53(19)$ \\
\hline $\mathrm{C} 3-\mathrm{C} 2-\mathrm{C} 5-\mathrm{O} 3$ & $-7.1(3)$ & $\mathrm{O} 2-\mathrm{C} 4-\mathrm{N} 2-\mathrm{C} 1$ & $-174.72(16)$ \\
\hline $\mathrm{C} 1-\mathrm{C} 2-\mathrm{C} 5-\mathrm{O} 3$ & $172.38(18)$ & $\mathrm{N} 1-\mathrm{C} 4-\mathrm{N} 2-\mathrm{C} 1$ & $5.0(2)$ \\
\hline $\mathrm{C} 2-\mathrm{C} 3-\mathrm{N} 1-\mathrm{C} 4$ & $1.3(2)$ & $\mathrm{O} 1-\mathrm{C} 1-\mathrm{N} 2-\mathrm{C} 4$ & $177.47(15)$ \\
\hline $\mathrm{C} 2-\mathrm{C} 3-\mathrm{N} 1-\mathrm{C} 6$ & $-178.27(15)$ & $\mathrm{C} 2-\mathrm{C} 1-\mathrm{N} 2-\mathrm{C} 4$ & $-2.7(2)$ \\
\hline
\end{tabular}

Hydrogen-bond geometry $\left(\AA,{ }^{\circ}\right)$

\begin{tabular}{lllll}
\hline$D-\mathrm{H} \cdots A$ & $D-\mathrm{H}$ & $\mathrm{H} \cdots A$ & $D \cdots A$ & $D-\mathrm{H} \cdots A$ \\
\hline $\mathrm{N} 2-\mathrm{H} 2 \cdots \mathrm{O} 1^{\mathrm{i}}$ & 0.86 & 1.98 & $2.8329(18)$ & 174 \\
\hline
\end{tabular}

Symmetry code: (i) $-x+2,-y+2,-z+1$. 